\title{
A Comparison of Neonatal Outreach Service Activity Before and During The First UK Wave of SARS-COV- 2 in Leicestershire, UK
}

\section{Vikas Saxena}

University Hospitals of Leicester NHS Trust

Claire Inglis

University Hospitals of Leicester NHS Trust

\section{Sumit Mittal}

University Hospitals of Leicester NHS Trust

Joe Fawke ( $\nabla$ joe.fawke@uhl-tr.nhs.uk)

University Hospitals of Leicester NHS Trust https://orcid.org/0000-0002-5267-7107

\section{Short Report}

Keywords: Infant, newborn, Bronchopulmonary dysplasia, Community outreach, SARS-COV-2, Feeding, tube

Posted Date: April 13th, 2021

DOI: https://doi.org/10.21203/rs.3.rs-396713/v1

License: (c) (i) This work is licensed under a Creative Commons Attribution 4.0 International License. Read Full License 


\section{Abstract}

Neonatal outreach services provide post discharge support to babies with additional needs and their families. This includes support for nasogastric feeding and supplemental home oxygen use for babies with bronchopulmonary dysplasia (BPD). People potentially at higher risk of serious COVID-19 infection have been encouraged to shield and vulnerable babies requiring supplemental oxygen were in this category.

This study reviews the impact of COVID-19 on a tertiary Neonatal service outreach team during the first UK COVID wave and lockdown in March-May 2020 compared to March-May 2019. Outreach service activity was captured on a team activity database including home visits, phone contacts, babies on home oxygen and overnight saturation monitoring.

Outreach home visits increased by $33 \%$, telephone contacts increased by $24 \%$ and additional support was required by families with 20 virtual clinics instead of 7 face to face clinics. However, emergency department attendances by babies receiving supplemental home oxygen dropped significantly. Emergency department attendances for these babies were over $700 \%$ higher pre-Covid than during this Covid lockdown period ( 1.8 vs 0.25 attendances / baby in home oxygen).

It was possible to maintain neonatal outreach support with adapted working including risk assessments and appropriate PPE. Feedback on the neonatal outreach service provision during Covid lockdown was received from $75 \%$ families with babies in supplemental home oxygen; $83 \%$ of them strongly agreed and $17 \%$ agreed that a high quality of care was provided during COVID-19. All respondents were extremely likely to recommend the service to friends and families.

\section{Introduction}

Neonatal outreach services provide post discharge support to babies with additional needs and their families. This can include support for nasogastric feeding and supplemental home oxygen use for babies with bronchopulmonary dysplasia (BPD).

The UK National Neonatal Audit project reported that between 2016 and 2018, 36.5\% (8,671 of 23,773) of babies born $<32$ weeks gestation had significant BPD or died before 36 weeks corrected gestational age [1]. Some infants with BPD are discharged on supplemental home oxygen and these babies have increased vulnerability to respiratory infections and higher hospital readmission rates [2] as well as increased parental anxiety [3]. After discharge supplemental oxygen is slowly weaned in the community, a process that requires careful monitoring of these vulnerable infants. Our neonatal outreach service provides community support through regular home visits, telephone contact, growth assessments, feeding advice and support during supplemental home oxygen weaning [4].

People potentially at higher risk of serious COVID-19 infection have been encouraged to shield and vulnerable babies requiring supplemental oxygen would fall into this category. Fortunately, initial 
concerns that SARS-COV-2 infection might be more severe in preterm babies were not realised, with no deaths attributable to SARS-CoV-2 reported in neonates in the UK between March and April, 2020 [5]. However, this information was not available during the March-May 2020 study period and parental and staff anxiety was high.

Of necessity national and international guidance has evolved but regular changes contribute to the anxiety felt by both families and healthcare staff. This is compounded by concerns that new variants of the virus may be more transmissible or more dangerous and if so whether this disproportionately affects vulnerable groups.

Community services were slowed down by evolving guidance around public health, PPE and infection control, higher sickness rates and the need for some staff to self-isolate or shield further reducing staffing levels. Geographical variation in advice as local outbreaks are tackled has affected several areas of the UK, notably Leicester.

\section{Methods}

The University Hospitals Leicester neonatal outreach team provides assistance with discharge planning and post discharge support for infants with ongoing health needs in the East Midlands South region. This includes teaching parents extended skills (e.g. nasogastric-tube feeding, basic life support) to safely care for their babies at home rather than in hospital which allows earlier safe discharge.

We compared the outreach team's overall activity and post-discharge support for infants discharged over two time periods; March to May of 2019 vs March to May of 2020. Outreach service activity was captured on a team activity database including home visits, phone contacts, babies on home oxygen and overnight saturation monitoring. Clinical data collected during visits was documented on the UK wide Badgernet database. Babies requiring out of hours emergency care were directed to the paediatric emergency department. Data on emergency department attendances were obtained from the University Hospitals Leicester Integrated Clinical Environment database.

A risk assessment tool for COVID-19 was used prior to each home visit to assess risk to staff and to prevent cross contamination between visits. If the risk was assessed to be high a phone conversation was provided instead of a face to face visit. For home visits, standard operating procedures for PPE were introduced which presumed that every household potentially could be infected with COVID-19. The outreach team used a feedback form to assess parental satisfaction with the service for babies in supplemental home oxygen during COVID-19; these forms were returned by post.

\section{Results}

Compared to March-May 2019, 7\% more babies (114 vs 122 babies) were discharged with outreach team support in March-May 2020. However, home visits increased by 33\% (427 vs 568) and telephone contacts 
increased by $24 \%$ (782 vs 969) (Table 1 ). This was achieved despite one full-time member of staff shielding, resulting in a $10 \%$ reduction of work force.

Between March and May 2019, 11 babies were discharged with supplemental home oxygen compared to 16 in March to May 2020. The amount of supplemental oxygen babies were discharged in was comparable (2019 range $0.1-0.4$ litres/min, mean $0.22 ; 2020$ range $0.1-0.5$ litres $/ \mathrm{min}$, mean 0.24 ).

There was a significant drop in emergency department presentations amongst babies discharged in supplemental home oxygen during COVID-19. In March to May 2019, 11 babies generated 20 presentations ( 1.8 presentations/baby) vs 4 presentations from 20 babies in 2020 ( 0.25 presentations/baby). The reasons for attending were mainly increased work of breathing and poor feeding.

Babies discharged in supplemental home oxygen received an average of 5.45 (2019) vs 4.75 (2020) overnight saturation recordings per baby in 3 months (Table 1). With overnight oxygen saturation monitoring 5 infants were weaned off supplemental home oxygen in the 2020 study period compared to 3 in the 2019 study period.

The outreach team successfully monitored growth and development and shared data via the UK wide Badgernet database to facilitate virtual multi-disciplinary BPD clinics. The number of clinics held for close monitoring significantly increased from 7 (all face to face) to 20 (all virtual).

Feedback was received from 12/16 families with babies in supplemental home oxygen; 10 of them strongly agreed and 2 agreed that a high quality of care was provided during COVID-19. 100\% of respondents were extremely likely to recommend the service to friends and families.

\section{Discussion}

During the UK COVID-19 first wave, when all NHS non-urgent services were being closed and resources diverted to meet the unprecedented demand on acute services, Neonatal Outreach services were maintained in Leicester and continued to work in the community using appropriate PPE and social distancing. A full range of services continued including looking after babies on supplemental home oxygen and those discharged with community support for nasogastric tube feeding. There was a lot of anxiety among parents and concern that unclear symptoms were due to COVID-19. This led to increased telephone calls for advice and requests for home visits to support this vulnerable population of infants and their families.

Most of the parents were self-isolating at home, in view of high-risk infants and some due to anxiety or suspected exposure. Isolation and social distancing disrupted family support networks. COVID-19 related financial stresses can compound mental health issues and overall increased stress levels have raised concerns about safeguarding and domestic violence issues nationally. Parental physical and mental health has been recognised as a significant factor in the care of children [4]. The need for support 
increased and this gap was in part filled by the neonatal outreach team whose perception was that worries were dealt with and reduced by their increased support.

The significant drop in emergency department presentations by babies in supplemental home oxygen may be because clinical issues were dealt with in the community or because parents were frightened to come to hospital due to the risk of contracting SARS-COV-2. The reduction in ED attendances was not associated with adverse health outcomes.

Our team worked at full capacity despite being affected by sickness and anxiety among team members in relation to home visits. Using a COVID-19 risk assessment before each home visit reduced the risk to staff members, reduced staff anxiety and protected infants. Outreach services collected anthropometry parameters during their routine visit to facilitate multi-disciplinary virtual clinics. These measurements would usually be done by a health visitor or midwife post discharge. Collection by the outreach ream reduced the need for other health care workers to visit to home and facilitated virtual clinics but did result in the visits taking longer.

It is well documented that parental anxiety results in increased utilisation of health services [6] we noted the number of telephone calls and home visits during COVID increased. Although parental anxiety was evident we did not use standardised tools to assess the level of parental anxiety. The impact of higher risk family members at home e.g. elderly relatives, higher risk ethnicities or those with comorbidities was not assessed.

\section{Conclusion}

Covid-19 changed the outreach teams working with increases in home visits and telephone support, increased growth monitoring and sharing of information to support virtual clinics. A combination of support and anxiety about attending hospital dramatically reduced emergency department presentations. Services were maintained despite staff anxieties, sickness and the challenges of infection prevention measures and PPE. Parents appreciated the input of the neonatal outreach team and rated the service highly.

\section{References}

1. National Neonatal Audit Programme (NNAP) 2019 annual report on 2018 data. RCPCH: London, 2019, RCPCH Resources NNAP Report https://www.rcpch.ac.uk/resources/national-neonatal-auditprogramme-annual-report-2019

2. Underwood MA, Danielsen B, Gilbert WM (2007) Cost, causes and rates of rehospitalisation of preterm infants. J Perinatol 27:614-619. DOI:10.1038/sj.jp.7211801

3. Zanardo V, Freato F, Home oxygen therapy in infants with bronchopulmonary dysplasia: assessment of parental anxiety, Early Human Development Volume 65, Issue 1, October 2001, Pages 39-46 DOI: 10.1016/s0378-3782(01)00190-6 
4. Balfour-Lynn IM, Field DJ, Gringras P et al (2009) BTS guidelines for home oxygen in children. Thorax 64:ii1-ii26. DOI:10.1136/thx.2009.116020

5. Gale C, Quigley MA, Placzek A, Knight M, Ladhani S, Draper ES et al (2021) Characteristics and outcomes of neonatal SARS-CoV-2 in the United Kingdom: a prospective national cohort study using active surveillance. Lancet Child Adolesc Health 5:113-121. doi:10.1016/S2352-4642(20)30342-4

6. Lee Goldman S, Owen MT (1994) The impact of parental trait anxiety on the utilization of health care services in infancy: a prospective study. Journal of Paediatric Psychology 19(3):369-381. DOI:10.1093/jpepsy/19.3.369

\section{Table}




\begin{tabular}{|c|c|c|c|}
\hline \multirow[t]{2}{*}{ Year ( March - May) } & & 2019 & 2020 \\
\hline & & & (percentage increase) \\
\hline \multirow[t]{3}{*}{ Total number of Home visits } & March & 132 & $198(33 \%)$ \\
\hline & April & 154 & $175(12 \%)$ \\
\hline & May & 141 & $195(27 \%)$ \\
\hline \multirow[t]{3}{*}{ Total number of calls received } & March & 198 & $281(29 \%)$ \\
\hline & April & 317 & $334(5 \%)$ \\
\hline & May & 267 & $354(24 \%)$ \\
\hline \multicolumn{4}{|c|}{ Services provided to vulnerable babies on Home Oxygen during Pandemic } \\
\hline \multicolumn{2}{|l|}{ Year (March - May) } & 2019 & 2020 \\
\hline \multicolumn{2}{|l|}{ Number of babies on home oxygen } & 11 & 16 \\
\hline \multicolumn{2}{|l|}{ Number of ED visits } & 20 & 4 \\
\hline \multicolumn{2}{|l|}{ Number of ED visits / baby in home oxygen } & 1.8 & $0.25^{\star}$ \\
\hline \multirow[t]{3}{*}{ Number of home visits for BPD patients } & March & 48 & 69 \\
\hline & April & 48 & 58 \\
\hline & May & 57 & 77 \\
\hline \multicolumn{2}{|l|}{ Total Number of home visits } & 153 & 204 \\
\hline \multicolumn{2}{|l|}{ Number of visits per baby per month } & 5.45 & 4.75 \\
\hline \multicolumn{2}{|l|}{ Number of overnight saturation studies } & 60 & 76 \\
\hline \multicolumn{2}{|l|}{ Number of babies taken off Home Oxygen } & 3 & 5 \\
\hline \multicolumn{2}{|l|}{ Number of BPD clinics } & 7 (all face to face) & 20 (all virtual)* \\
\hline
\end{tabular}

